\title{
An Inventory Solution Model for down and up Processing and Profit with Scrap Clearing in Fuzzy Sense
}

\author{
N. Nalini ${ }^{1 *}$ and E. Chandrasekaran ${ }^{2}$ \\ 'Mathematics Department, Presidency College/University of Madras, Chennai - 600005, Tamil Nadu, India; \\ nrkhome@yahoo.com \\ ${ }^{2}$ Vel Tech University, Chennai - 600062, Tamil Nadu, India; \\ e_chandrasekaran@yahoo.com
}

\begin{abstract}
Objectives: To develop fuzzy set theory and the fuzziness in the inventory problem, the purpose is to find the Revenue for defective quantity corresponds to the total cost and the associated cost of selling value of remodeled product also fuzzy. Methods/Statistical Analysis: This approach is to find the quantity which has the minimum cost with maximum profit. When the profit gained from selling one unit of the item with warranty periods and guaranty period Up process cost and other scrap cost must be gives some percentage of profits. Partial profit of planned operation on stock clearing and effective scrap clearing of the last products has to be done. Findings: Mathematical model has been developed in below ways, 1 . To find the fuzzy down process with the fuzzy relevant cost, 2 . Revenue related with fuzzy production cost and profit of the different lot sizes of various products, 3 . To find the fuzzy up process with the fuzzy screening cost of defective quantity with time dependent relevant cost tends to profits with partial loss and 4 . Effective scrap clearing and total profit of all the goods. Applications/Improvements: Our aim is to find total cost and profit with fuzzy sense. Numerical examples are given and sensitivity analysis is carried out to conclude the result.
\end{abstract}

Keywords: Down Process, Fuzzy Profit, Relevant Cost, Revenue, Screening Cost, Trapezoidal Fuzzy Numbers, Up Process

\section{Introduction}

The manufacturer had developed a new generation gadgets which is different from the older gadgets. Some ideas observed from used items were reusable in future products. This process in which used components must upgraded and modified to satisfy the exactly same quality or higher quality of new generation products in remodeled way. Companies are applying remaking is the ability to attract more customers due to environment and pocket friendly prices.

The defective items with immediate return analyzed and found the solution of ranking method with fuzzified purchase cost and relevant cost with triangular fuzzy number ${ }^{1}$. The trapezoidal fuzzy demand and additional demand pattern with shortages and backlogged condition $^{2}$. Defective items sold after screening process and deep on that, mostly in trade returns goods goes to manufacturing only. But what is the use of that? Relevant cost and sending the defectives are waste of money, but proper planning for defectives will make an additional profit with partial Loss ${ }^{3}$, developed a trade credit model with multivariable demand and the objective of review the procedure with multivariate demand under diminishing conditions has been solved ${ }^{4}$. Mathematical model for Economic Order Quantity model with immediate return of defective items ${ }^{5}$. A classic EOQ model with fixed values and purchase, relevant costs are added with discounts and partial deterioration costs ${ }^{6}$. Ordering, holding cost and safety stock with fuzzy arithmetic operators ${ }^{7}$.Wellknown

*Author for correspondence 
economic order quantity model demand with permissible delay in payments with solutions analysed ${ }^{8}$. Basic theory of operators and multiple lot size and preplanned profitable return of goods ${ }^{9,10}$. The partial backlogging inventory model for deteriorating items considering stock and price sensitive demand rate in fuzzy sense ${ }^{11}$. A multi-item inventory model of deteriorating items with expiration date is developed and analyzed ${ }^{12}$. Recent trends and comparison of various authors in inventory control strategy ${ }^{13}$. Determined the optimum production cycle which tends to reduce the total cost of the inventory system ${ }^{14}$. To maximize the resultant profit and it contain with demand and reordering process ${ }^{15}$. How to reduce cost of recruitment, advertisement, holding, travel allowance and management cost are discussed ${ }^{16}$. Partial backlogging and deterioration governs with differential equations ${ }^{17}$. Considering an optimal inventory model for deteriorating items and demand rate depends price and solved the model for total profit ${ }^{18}$. Left and right triangular fuzzy numbers used to find relevant cost and return of goods $\operatorname{cost}^{19}$. Developed two stages scheduling for to minimize rental cost under fuzzy time using triangular fuzzy number can be extended to trapezoidal fuzzy numbers with different parameters ${ }^{20}$. Elementary applications on operations on generalized trapezoidal fuzzy numbers ${ }^{21}$. Operations on trapezoidal fuzzy numbers with uncertain conditions ${ }^{22}$. Defective item solution depends with constant demand and time developed ${ }^{23}$. The standard of manufacturing technique is to obtain optimal fuzzy rule ${ }^{24} .{ }^{25}$ Analysed fuzzy trapezoidal rule for average mean value method. Fuzzy optimization plays the main role in data mining and share market tradings ${ }^{26,27}$.

The length of process for replacement in the main gadgets for determining profit and it has been found they no longer the waiting time, well planned and prepared team work is lower the repair rate. Customers who experience ensure quality may out purchase the goods again from the respective manufacturer and they may turn to another brand to purchase consequently a larger quantity of sales is lost, tends to a smaller profit. In fact partial loss factor should be avoided and profit also be fuzzy in nature. However the real life inventory problems can be properly formulated in this way due to expected loss includes scrap revenue also.

\subsection{Up and Down Process}

This process is comparison of products with direct profit and reworked products with indirect profit, addition of this two we will get total profit. Formulation of mathematical model, in inventory problems there are generally multiple objectives. The model is to determine the holding, ordering and purchasing in order to keep the total relevant cost as low as possible. Profit is addition of product selling revenue and returned products revenue also. In addition to that well planned scrap clearing section also gives the revenue. Three different processes have to do, one is direct selling products without any complaints and other one is repairable or serviceable products, so we will get two demands, actual demand and company second sale demand. Good products are get ready with earlier way to the market or repairable goods are late to market with some profit. If we are handling two things in a system it is called mixed system with two process, one is Down process it is used for Good Quality First Sale (GQFS) and second one Up process is Defective Quality Second Sale (DQSS) and remaining defective products goes to scrap clearing section.

First section is materials and methods of the findings, second section is two process are depends Up and Down Process. Third is the mathematical models in fuzzy environment has to solve with Up and Down process. Fourth, five and six section explanation of numerical examples for process is comparison of products with direct profit and return of products with indirect profit, addition of this two we will get total profit and actions should be taken for increase the ownership costs scrap clearing cost also included. Finally section seven, conclusion and future research.

\section{Notations and Assumptions}

$\sim$ wavy bar indicates fuzzification of parameters.

$\widetilde{\text { DP }}$ is the Down Process.

$\widetilde{\text { UP }}$ is the Up Process.

$\widetilde{\mathbf{S P}}$ is the Scarp Process.

$\widetilde{\mathbf{D}_{\mathrm{q}}}$ is the Fuzzy Demand quantity.

$\widetilde{\mathbf{H}}$ is the Holding cost

$\widetilde{\mathbf{O}}$ is the Ordering or setup cost

$\widetilde{r}$ is the Order quantity in Down Process

$\widetilde{r}^{*}$ is the Order quantity in Up Process

$\tilde{\mathbf{r}}^{* *}$ is the Order quantity in $\frac{\text { Clearing }}{\text { recycling }}$ Process

$\widetilde{L_{r}}$ is the Length of the plan. 
$\widetilde{L_{1}}$ is the Length of the plan in Down process (90 days). $\widetilde{L_{\mathbf{2}}}$ is the Length of the plan in Up process (30 days).

$L_{\mathbf{3}}$ is the Length of the plan in clearing process $(30$ days).

$\mathrm{D}_{\mathrm{f}}$ is the percentage of defective items.

Let $\tilde{r}=\left(1-D_{f} \%\right)$ is the quantity of good items.

$\tilde{r}^{*}=\left(D_{f}\right)$ is the defective quantity, here, $D_{f}=a+b$

$\tilde{r}^{*}=b$ is the clearing / scrap quantity.

Assume,

$D_{f}(\alpha, \beta, \gamma, \delta)=(5,10,15,20) \%$

$\mathrm{R}_{1}$ is the Testing and dismantling (depreciation cost).

$\mathrm{R}_{2}$ is the Labor cost.

$\mathrm{R}_{3}$ is the Additional Material cost/ Upgrading and quality test cost.

$\mathrm{R}_{4}$ is the Scrap cost.

$\mathrm{Cc}$ is the Clearing Charges.

$S \widetilde{V}_{G}$ is the Selling value of good items in down process per unit

$S \widetilde{V}_{D}$ is the Selling value of Defective items in Up process per unit

$S \widetilde{V}_{c}$ is the Selling value of Scrap items in clearing section per unit

$\left[R_{\text {ev1 }} D N\right]$ is the Revenue in Down Process

$\left[R_{\mathrm{ev} 2} \mathbf{U P}\right]$ is the Revenue in Up Process

$\left[\widehat{R_{e v} R c}\right]$ is the Revenue in Recycling Process

$\left[\widehat{P_{1} D N}\right]$ is the gain in Down Process

$\left[\widehat{P_{2} U P}\right]$ is the gain in Up Process

$\left[\widehat{P_{3} R c}\right]$ is the gain in $\frac{\text { Recycling }}{\text { scrap }}$ Process

[TDN] is the Total Cost in Down Process

[TUP] is the Total cost in UP Process

[ $\widehat{\text { TRC] }}$ is Total cost of Recycling/scrap Process

3. Mathematical Model Formulations

3.1 To find Purchase Cost

Let $\widetilde{\mathbf{P}_{\mathrm{Uc}}}=\left(\widetilde{\mathbf{P}_{\mathrm{U} 1}}, \widetilde{\mathbf{P}_{\mathrm{U} 2}}, \widetilde{\mathbf{P}_{\mathrm{U} 3}}, \widetilde{\mathbf{P}_{\mathrm{U} 4}}\right)$ and $\tilde{\mathbf{r}}=\left(\widetilde{\mathbf{r}_{1}}, \widetilde{\mathbf{r}_{2}}, \widetilde{\mathbf{r}_{3}}, \widetilde{\mathbf{r}_{4}}\right)$ are Trapezoidal fuzzy numbers, then

Purchasing Cost in Fuzzy Sense $=$ Purchase cost per unit $\times$ order quantity

$$
\begin{gathered}
{[\widetilde{P C}]=\widetilde{\mathrm{P}_{\mathrm{Uc}}} \otimes \tilde{\mathrm{r}}} \\
{[\widetilde{P C}]=\left(\widetilde{\mathrm{P}_{\mathrm{U} 1}}, \widetilde{\mathrm{P}_{\mathrm{U} 2}}, \widetilde{\mathrm{P}_{\mathrm{U} 3}}, \widetilde{\mathrm{P}_{\mathrm{U} 4}}\right) \otimes\left(\widetilde{\mathrm{r}_{1}}, \widetilde{\mathrm{r}_{2}}, \widetilde{\mathrm{r}_{3}}, \widetilde{\mathrm{r}_{4}}\right)} \\
{[\widetilde{P C}]=\left(\widetilde{\mathrm{P}_{\mathrm{U} 1}} \otimes \widetilde{\mathrm{r}_{1}}, \widetilde{\mathrm{P}_{\mathrm{U} 2}} \otimes \widetilde{\mathrm{r}_{1}}, \widetilde{\mathrm{P}_{\mathrm{U} 3}} \otimes \widetilde{\mathrm{r}_{1}}, \widetilde{\mathrm{P}_{\mathrm{U} 4}} \otimes \widetilde{\mathrm{r}_{1}}\right)}
\end{gathered}
$$

Holding Cost in Fuzzy Sense $=\widetilde{\mathbf{H}} \otimes \widetilde{\mathbf{r}} \otimes \frac{\widetilde{\mathrm{L}}_{\mathrm{r}}}{2}$

$$
\text { Ordering cost in fuzzy sense }=\sigma \otimes \frac{\sigma_{q}}{r^{r}}
$$

Total cost is addition of holding cost and Setup cost ${ }^{1}$,

Let. $\widetilde{\mathbf{P}_{\mathrm{Uc}}}=\left(\widetilde{\mathrm{P}_{\mathrm{U} 1}}, \widetilde{\mathrm{P}_{\mathrm{U} 2}}, \widetilde{\mathrm{P}_{\mathrm{U} 3}}, \widetilde{\mathrm{P}_{\mathrm{U} 4}}\right), \tilde{\mathbf{r}}=\left(\widetilde{\mathbf{r}_{1}}, \widetilde{\mathbf{r}_{2}}, \widetilde{\mathbf{r}_{3}}, \widetilde{\mathbf{r}_{4}}\right)$ are Trapezoidal Fuzzy Numbers 21, 22, Then, From Equations (1), (2) and (3),

Total cost in fuzzy $=\left[\tilde{P}_{\mathrm{Uc}} \quad \otimes \tilde{\mathrm{r}}\right] \oplus\left[\tilde{\mathrm{H}} \otimes \tilde{\mathrm{r}} \frac{\otimes \tilde{\mathrm{L}}_{\mathrm{r}}}{2}\right] \oplus\left[\tilde{\mathrm{O}} \otimes \frac{\widetilde{\mathrm{D}_{q}}}{\tilde{\mathrm{r}}}\right](4)$

\subsection{To find Total Cost in Down Process}

Let $D=\left(\widetilde{D_{1}}, \widetilde{\mathbf{D}_{2}}, \widetilde{\mathbf{D}_{3}}, \widetilde{\mathbf{D}_{4}}\right)$,

$\widetilde{H}=\left(\widetilde{H_{1}}, \widetilde{\mathrm{H}_{2}}, \widetilde{\mathrm{H}_{3}}, \widetilde{\mathrm{H}_{4}}\right), \widetilde{O}=\left(\widetilde{O_{1}}, \widetilde{\mathrm{O}_{2}}, \widetilde{\mathrm{O}_{3}}, \widetilde{\mathrm{O}_{4}}\right)$

are trapezoidal fuzzy numbers 21,22 , then,

$$
\begin{aligned}
& {[\widetilde{T D N}]=\left[\widetilde{\mathbf{P}_{\mathrm{Uc}}} \otimes \tilde{\mathbf{r}}\right] \oplus\left[\widetilde{H} \otimes \widetilde{r} \frac{\otimes \widetilde{L_{r}}}{2} \oplus \widetilde{O} \otimes \frac{\widetilde{D_{q}}}{\tilde{r}}\right]} \\
& =\left[\left[\left(\left(\mathrm{P}_{\downarrow} \mathrm{U} 1\right)^{*},\left(\mathrm{P}_{\downarrow} \mathrm{U} 2\right)^{*},\left(\mathrm{P}_{\downarrow} \mathrm{U} 3\right)^{*},\left(\mathrm{P}_{\downarrow} \mathrm{U} 4\right)^{*}\right) \otimes\right.\right. \\
& \left.\left(\left(r_{\downarrow} 1\right)^{\sim},\left(r_{\downarrow} 2\right)^{\sim},\left(r_{\downarrow} 3\right)^{\sim},\left(r_{\downarrow} 4\right)^{\sim}\right)\right] \oplus[\text { [ EMBED }
\end{aligned}
$$

Equation. 3 园圆圆 $\otimes(r)^{-} \otimes\left(\square(L)^{-}{ }_{\downarrow} 1,\left(L_{\downarrow} 2,\right)^{-}\right.$

$$
\left.\left.\left(L_{\downarrow} 3,\right)^{-}\left(L_{\downarrow} 4\right)^{\sim}\right) \oslash 2\right] \oplus\left(\left(O_{\downarrow} 1,\right)^{-}\left(O_{\downarrow} 2,\right)^{-}(\right.
$$

$[\widetilde{T D N}]=\left[\widetilde{\mathrm{P}_{\mathrm{U} 1}} \otimes \widetilde{\mathrm{r}_{1}}, \widetilde{\mathrm{P}_{\mathrm{U} 2}} \otimes \widetilde{\mathrm{r}_{1}}, \widetilde{\mathrm{P}_{\mathrm{U} 3}} \otimes \widetilde{\mathrm{r}_{1}}, \widetilde{\mathrm{P}_{\mathrm{U}}} \otimes \widetilde{\mathrm{r}_{1}}\right] \oplus$

$$
\left[\widetilde{H_{1}} \otimes \widetilde{r} \otimes \frac{\widetilde{L}_{1}}{2}, \widetilde{H_{2}} \otimes \widetilde{r} \frac{\otimes \widetilde{L}_{2}}{2}, \widetilde{H}_{3} \otimes \widetilde{r} \frac{\otimes \widetilde{L}_{3}}{2}, \widetilde{H}_{3}\right.
$$

$$
\left.\otimes \widetilde{r} \oplus \frac{\widetilde{L_{4}}}{2}\right] \oplus\left[\widetilde{O_{1}} \otimes \frac{\widetilde{D_{1}}}{\tilde{r}}, \widetilde{O_{2}} \otimes \frac{\widetilde{D_{2}}}{\widetilde{r} O_{3}} \otimes \frac{\widetilde{D_{3}}}{\widetilde{r, O_{4}}} \otimes \frac{\widetilde{\sigma_{4}}}{\tilde{r}}\right]
$$

$$
\mathrm{O}_{3}
$$

$$
\begin{aligned}
& \text { Let } \tilde{r}=\left(1-D_{f \mathbf{x}}\right) \tilde{r}=\left(1-D_{f \mathbf{x}}\right) \\
& \tilde{r}^{*}=\left(D_{f \star}\right) \text { here, } D_{f \star x}=a+b \\
& \tilde{r}^{*}=b
\end{aligned}
$$

Repairable and recyclable scrap and Up process total cost $=$ Holding cost + Testing and Dismantling (depreciation cost) + Labor cost + Additional Material cost/ Upgrading and quality test cost + Scrap cost + Clearing charges ${ }^{1}$. 


\subsection{To find Total Cost in Up Process}

Total cost in Up process $=$ Holding cost + Testing and Dismantling cost + Labor cost + Additional Material cost/ Upgrading and quality test cost ${ }^{1}$.

$[\widetilde{T U P}]=\left[\widetilde{H} \otimes \widetilde{r^{*}} \frac{\widetilde{L}_{2}}{2}\right] \oplus\left[\left(R_{1} \oplus \widetilde{R_{2}}\right) \otimes\left(r^{*}\right]\right)$

$\oplus\left[\left(R_{3}\right) \otimes\left(\widetilde{r^{8}} \sim \widetilde{r^{* 8}}\right)\right]$

$=\left[\right.$ EMBED Equation. 3 目目目 $\left.\otimes \widetilde{r^{*}} \frac{\otimes \widetilde{L_{2}}}{2}\right]$

$\oplus\left[\left(R_{1} \oplus R_{2}\right) \otimes D_{f \times}\right] \oplus\left[\left(R_{3}\right) \otimes\left(r^{*} \Theta b\right)\right]$

$=\left[\left(H_{\downarrow} 1\right)^{-} \otimes\left(r^{\uparrow} *\right)^{2} \square \otimes(L)^{-}-\downarrow \downarrow 2,\left(H_{\downarrow} 2\right)^{-}\right.$

$\otimes\left(r^{\uparrow} *\right)^{2} \square \otimes(L)^{-} \square_{1} 202,\left(H_{\downarrow} 3\right)^{-} \otimes\left(r^{\top} *\right)^{-}$

$\Theta(L)_{-} 202,\left(H_{\downarrow} 4\right)^{-} \otimes\left(r^{\dagger} *\right)^{-} \otimes(L)^{-} 2$

(D) 2] $\oplus\left[\left(R_{\downarrow} 1 \oplus R_{\downarrow} 2\right) \otimes D_{\downarrow}(f \%)\right] \oplus\left[\left(R_{\downarrow} 3\right) \otimes(a)\right.$

$r^{*}$

$r^{*}$

$r^{*}$

3.4 To find the Total Cost of Recycling/ Clearing Scrap Process

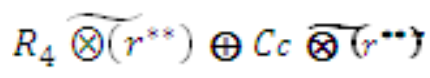

$=\left[\left(H_{\downarrow} 1\right)^{-} \otimes\left(r^{\top}(* *)\right)^{-} \otimes(L)^{-} \tau_{1} 302\right.$,

$$
\left(H_{1} 2\right)^{\sim} \otimes\left(r^{\top}(* *)\right)^{-\top} \otimes(L)^{-}-_{1} 302,
$$

$\left(H_{1} 3\right)^{\sim} \otimes\left(r^{\dagger}(* *)\right)^{-} \otimes(L)^{2} \square_{1} 302,\left(H_{1} 4\right)$

$\left.\because\left(r^{\dagger}(* *)\right)^{-\square} \otimes(L)^{2} \square_{1} 3 \oslash 2\right] \oplus\left[\left(R_{1} 4\right.\right.$

$$
\left.\otimes\left(r^{\dagger}(* *)\right)\right)^{-} \oplus\left(C c \otimes\left(r^{\dagger}(* *)\right)^{*}\right)
$$

$$
\left[(T R C)^{-}\right]=\left[\left(H_{1} 1\right)^{\sim} \otimes\left(r^{\dagger}(* *)\right)^{-\top} \otimes(L)^{-} \varpi_{1} 3\right.
$$

$\emptyset 2 \oplus\left[\left(R_{1} 4 \otimes\left(r^{\dagger}(* *)\right)\right)^{-} \oplus\left(C C \otimes\left(r^{\dagger}(* *)\right)^{*}\right)\right]$, $\left(H_{\downarrow} 2\right)^{-} \otimes\left(r^{\uparrow}(* *)\right)^{2} \square \otimes L \nabla \downarrow 302 \oplus\left[\left(R_{\downarrow} 4\right.\right.$

$$
\begin{aligned}
& \left.\left.\otimes\left(r^{\dagger}(* *)\right)\right)^{-} \oplus\left(C c \otimes\left(r^{\dagger}(* *)\right)^{*}\right)\right],\left(H_{\downarrow} 3\right)^{\sim} \\
& \otimes\left(r^{\dagger}(* *)\right) \otimes(1 \\
& {[\widetilde{T R C}]} \\
& =\left[\left(H_{\downarrow} 1\right)^{-} \otimes\left(r^{\uparrow}(* *)\right)^{-} \square \otimes(L)^{\sim} \square_{\downarrow} 302\right. \\
& \oplus\left[\left(R_{\downarrow} 4 \otimes b\right) \oplus(C c \otimes b)\right],\left(H_{\downarrow} 2\right)^{-} \otimes \\
& \left(r^{\uparrow}(* *)\right)^{-} \otimes(L)^{-} 3 \emptyset 2 \oplus\left[\left(R_{\downarrow} 4\right.\right. \\
& (\otimes b) \oplus(C c \otimes b)],\left(H_{\downarrow} 3\right)^{\sim} \otimes\left(r^{\dagger}(* *)\right)^{\sim} \square \otimes \\
& (L)^{-} \square_{1} 02 \oplus\left[\left(R_{1} 4 \otimes b\right) \oplus(C c \otimes b)\right],
\end{aligned}
$$

\subsection{To find the Revenue in Down Process}

Let $\mathrm{V}=\mathrm{V}_{1}, \mathrm{~V}_{2}, \mathrm{~V}_{3}$ in three revenue periods up, down and Scrap clearing sections,

Let $r=\left(r_{1}, r_{2}, r_{3}, r_{4}\right)$ and $S \nabla_{G}=\left(S \nabla_{1}, S \nabla_{2}, S \nabla_{3}, S \nabla_{4}\right)$ are trapezoidal Fuzzy numbers then,

$\left[R_{e v} \widetilde{D} N\right]=$ Selling PriceXQuantity of items in Down process

$$
\begin{aligned}
{\left[R_{e v} \widetilde{D} N\right]=\left[\widetilde{S V_{G}} \otimes \widetilde{r}\right] } \\
=\left[\left(S \nabla_{1}, S V_{2}, S \nabla_{3}, S \nabla_{4}\right) \otimes\left(r_{1}, r_{2}, r_{3}, r_{4}\right)\right]
\end{aligned}
$$

$\left[R_{\ell V_{1} D} D\right]=\left[\left(\widetilde{S V_{1}} \otimes \widetilde{r}_{1}, \widetilde{S V_{2}} \otimes \tilde{r}_{2}, \widetilde{S V_{3}} \otimes \widetilde{r}_{3}, \widetilde{S V}_{4} \otimes \widetilde{r}_{4}\right)\right](9)$

\subsection{To find the Gain in Down Process}

Let $\mathrm{F}=\mathrm{F}_{1}, \mathrm{~F}_{2,} \mathrm{~F}_{3}$ are the profit in three places are Down, $\mathrm{Up}$ and Scrap clearing sections.

Profit $=$ Revenue - Total Cost $^{1}$

$\left[\widetilde{P_{F} \overline{D N}}\right]=\left[R_{\Theta v} \widetilde{D N}\right] \Theta[\widetilde{T \overline{D N}}]$

$\left(\left[P_{\downarrow} 1 D N\right]\right)^{-}=\left[\left[\left(-S V \square_{\downarrow} 1\right)^{-} \otimes r r_{\downarrow} 1,\left(\beth S V \square_{\downarrow}(2)\right)^{\prime}\right.\right.$

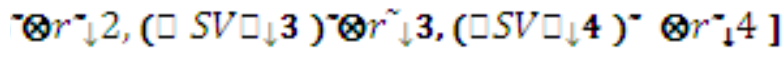

$\theta\left[\left(P_{\downarrow} U 1\right)^{-} \otimes\left(r_{\downarrow} 1\right)^{*} ;\left(P_{\downarrow} U 2\right)^{-} \otimes\left(r_{\downarrow} 1\right)^{*}\right.$;

$\left.\left(P_{\downarrow} \mathrm{U} 3\right)^{-} \otimes\left(\mathrm{r}_{\downarrow} 1\right)^{\sim},\left(\mathrm{P}_{\downarrow} \mathrm{U} 4\right)^{-} \otimes\left(\mathrm{r}_{\downarrow} 1\right)^{-}\right] \oplus$

$\left[\left(H_{\downarrow} 1\right) \otimes(r)^{*} \otimes \square(L)^{-} \square_{\downarrow} 1\right.$

$\{$ by the equation(5) and (9)\} 


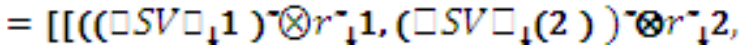

$\left.\left(\left[S V \square_{1} 3\right)^{-} \otimes r_{\downarrow}^{*} 3,\left(\square S V \square_{1} 4\right)^{-} \otimes r_{t}^{-} 4\right)\right] \Theta$

$\left[\left(P_{\downarrow} U 1\right)^{-} \otimes\left(r_{\downarrow} 1\right)^{*} ;\left(P_{\downarrow} U 2\right)^{-} \otimes\left(r_{\downarrow} 1\right)^{\sim},\left(P_{\downarrow} U 3\right)\right.$

$\left.\cdot \otimes\left(\mathrm{r}_{\downarrow} 1\right)^{\prime},\left(\mathrm{P}_{\downarrow} \mathrm{U} 4\right)^{-} \otimes\left(\mathrm{r}_{\downarrow} 1\right)^{-}\right] \oplus\left[\left(H_{\downarrow} 1\right)\right.$

$\bullet \otimes(r)^{-} \otimes \square(L)^{\sim} \square_{\downarrow} 1$

$\widetilde{S V_{1}} \otimes \tilde{r_{1}} \ominus \widetilde{P_{\mathrm{U}}} \otimes \widetilde{r_{1}} \oplus \widetilde{H_{1}} \otimes \widetilde{r} \otimes \frac{\widetilde{L_{1}}}{2} \oplus \widetilde{O_{1}}$

$\otimes \frac{\widetilde{D_{1}}}{\tilde{r}}, S \nabla_{2} \otimes r_{2} \ominus \widetilde{\mathrm{P}_{\mathrm{U} 2}} \otimes \widetilde{r_{2}} \oplus \widetilde{H_{2}} \otimes \widetilde{r_{2}} \frac{\otimes L_{2}}{2}$

$\oplus \widetilde{O_{2}} \otimes \frac{\widetilde{T_{2}}}{r}, \widetilde{\mathrm{P}_{\mathrm{U}} 3} \otimes \widetilde{\mathrm{r}_{3}} \oplus \widetilde{S V_{3}} \bigotimes \otimes$

$(10) \mathrm{t}$

3.7 To find the Revenue in Up Process

Let $r^{*}=\left(r_{1}^{*}, r_{2}^{*}, r_{3}^{*}, r_{4}^{*}\right)$ and $S \nabla_{D}=\left(S \pi_{1}, S \pi_{2}, S T_{3}, S T_{4}\right)$

are trapezoidal Fuzzy numbers then,

$\left[R_{\vartheta v} \widetilde{U} P\right]=$ Selling PriceXQuantity of items

$\left[R_{\varepsilon v} \widetilde{U} P\right]=\left[\widetilde{S V_{D}} \otimes \widetilde{r^{2}}\right]$

$=\left[\left(S \nabla_{1}, S \nabla_{2}, S \nabla_{3}, S T_{4}\right) \otimes\left(r_{1}^{*}, r_{2}^{*}, r_{3}^{*}, r_{4}^{*}\right)\right]$

$\left[R_{\varepsilon v 2} \widetilde{D} N\right]=\left[\left(\widetilde{S V_{1}} \otimes \widetilde{r}_{1}^{*}, \widetilde{S V_{2}} \otimes \tilde{r}_{2}^{*}, \overline{S V_{3}} \otimes \widetilde{r}_{3}^{*}, \widetilde{S V_{4}} \otimes \tilde{r}_{4}^{*}\right)\right]$

\subsection{To find the Gain in Up Process}

$\left[\widetilde{P_{F} \bar{U} P}\right]=\left[\widetilde{R_{v V}} \widetilde{U} P\right] \Theta[\widetilde{T U P}]$

$\left(R_{1} \oplus R_{\mathbf{2}}\right) \bigotimes \otimes\{$ by the Equation (7) and (11)\}

$\left(R_{1} \oplus R_{2}\right) \otimes D$

$\left(\left[P_{\downarrow} 2 U P\right]\right)^{\sim}=\left[\left(\left[S V \square_{\downarrow} 1\right)^{\sim} \otimes \square r_{\downarrow}^{\sim} 1^{-\dagger} * \ominus\left(H_{\downarrow} 1\right)^{\sim}\right.\right.$ $\otimes\left(r^{\dagger} *\right)^{-} \otimes(L)^{2} \square_{1} 2 \oslash 2 \oplus\left[\left(R_{\downarrow} 1 \oplus R_{\downarrow} 2\right) \otimes D_{\downarrow}\right.$ $(f \%)] \oplus\left[\left(R_{\downarrow} 3\right) \otimes(a)\right],\left(\left[S V \beth_{\perp}(2)\right)^{-} \otimes \square r_{\downarrow}^{-} 2 \square^{\uparrow}\right.$ $* \Theta\left(H_{\downarrow} 2\right)^{-} \otimes\left(r^{\dagger} *\right)^{-\tau} \otimes(L)^{-}-\downarrow 202 \oplus \oplus[$ $\left(R_{\downarrow} 1 \oplus R_{\downarrow} 2\right) \otimes D_{\downarrow}(f \%)$

\subsection{To find the Revenue in Clearing/}

Recycling Process

Let $r^{*}=\left(r_{1}^{* *}, r_{2}^{* *}, r_{3}^{* *}, r_{4}^{* *}\right)$ and $S \nabla_{c}=\left(S \nabla_{1}, S T_{2}, S \pi_{3}, S \pi_{4}\right)$

are trapezoidal Fuzzy numbers then,
$\left[\widetilde{R_{\varepsilon v} R} c\right]=$ Selling PriceXQuantity of items

$\left[R_{\varepsilon a} \widetilde{R} C\right]=\left[\widetilde{S V}_{C} \otimes r^{\nabla *}\right]$

$=\left[\left(S \nabla_{1}, S \nabla_{2}, S \nabla_{3}, S \nabla_{4}\right) \otimes\left(r_{1}^{* *}, r_{2}^{* *}, r_{3}^{* *}, r_{4}^{* *}\right)\right]$

$\left[R_{\varepsilon 8} \widetilde{R} C\right]=\left[\left(\widetilde{S V_{1}} \otimes \tilde{r}_{1}^{* *}, \widetilde{V_{2}} \otimes \tilde{r}_{2}^{* *}, \overline{S V_{3}} \otimes \tilde{r}_{3}^{*}, \widetilde{S V_{4}} \otimes \tilde{r}_{4}^{* *}\right)\right]$

3.10 To find the Gain in Recycling/Scrap Process

$\left[\widetilde{P_{F} \bar{R} C}\right]=\left[\widetilde{R_{e v}} \widetilde{R} C\right] \Theta[\widetilde{T \overrightarrow{R C}}]$

$\left(\left[P_{\downarrow} 3 R C\right]\right)^{*}=\left[\left[\left(\left(\square S V \square_{\downarrow} 1\right)^{\sim} \otimes \square r^{*}{ }^{-1} \square^{\dagger}(* *)\right.\right.\right.$,

$\left(\square S V \square_{1}(2)\right)^{-} \otimes{ }_{-} r_{1}^{*} 2 \square^{\uparrow}(* *),\left(\square S V \square_{\downarrow} 3\right)^{-} \otimes \square$

$\left.\left.r_{\downarrow}^{\sim} 3 \square^{\dagger}(* *),\left(\square S V \square_{\downarrow} 4\right)^{-} \otimes \square r_{\downarrow}^{*} 4 \square^{\dagger}(* *)\right)\right]$

$\Theta\left[\left(H_{1} 1\right)^{*} \otimes\left(r^{\uparrow}(* *)\right)^{*} \square \otimes(L)^{2} \square_{\downarrow} 3 \emptyset 2\right.$

$\oplus\left[\left(R_{\downarrow} 4 \otimes b\right) \oplus(\mathrm{Cc} \otimes b)\right],\left(H_{\downarrow} 2\right)^{\sim} \bigotimes_{\{b y}$ the

Equation (8) and (13)\}

$$
r+T
$$

Adding Equation (10), (12) and (14) we will get the total profit of the entire trade,

$[\widetilde{T P r}]=\left[\widetilde{P_{1} \overline{D N}}\right] \oplus\left[\widetilde{P_{2}} \widetilde{U P}\right] \oplus\left[\widetilde{P_{3} \overline{R C}}\right]$

$([T P r])^{-}=\left[\left(\left[S V \beth_{1} 1\right)^{-} \otimes r_{1}^{-} 1 \Theta\left(\mathbf{P}_{1} \mathbf{U} 1\right)^{-}\right.\right.$

$\otimes\left(\mathrm{r}_{\downarrow} 1\right)^{-} \oplus\left(H_{\downarrow} \mathbf{1}\right)^{-} \otimes(r)^{-} \otimes-(L)^{-} \square_{\downarrow} 1$

$\emptyset 2 \oplus\left(O_{\perp} 1\right)^{-} \otimes\left(D_{1} 1\right)^{-} \oslash r^{*},\left(\left[S V_{-}(2)\right)^{-\otimes}\right.$

$r_{\downarrow}^{*} 2 \Theta\left(\mathbf{P}_{\downarrow} \mathrm{U} 2\right)^{-} \otimes\left(\mathbf{r}_{\downarrow} 2\right)^{-} \oplus\left(H_{\downarrow} 2\right)$

$\because \otimes(r)=\otimes(L)^{\top} \beth_{\downarrow} 2 \emptyset 2 \oplus(O \downarrow 2) \otimes$

$\left[\left(\left(\square S V \square_{\downarrow} 1\right)^{-} \otimes \square r_{\downarrow}^{-} 1^{-\uparrow}(* *) \ominus\left(H_{\downarrow} 1\right)^{\sim}\right.\right.$

$\otimes\left(r^{\dagger}(* *)\right)^{\tau} \otimes(L)^{-\tau_{1}} 3 \emptyset 2 \oplus\left[\left(R_{1} 4 \otimes b\right)\right.$

$\left.\oplus(C c \otimes b)],\left(\square S V \square_{\perp}(2)\right)\right)^{-} \otimes \square r^{-}{ }^{-} \square^{\dagger}(* *) \Theta$

$\left(H_{\downarrow} 2\right)^{-} \otimes\left(r^{\dagger}(* *)\right)^{-\square} \otimes(L)^{2}-\downarrow 302 \oplus$ $\left[\left(R_{\downarrow} 4 \otimes b\right) \oplus(C c \otimes b)\right],(\square S V \square \downarrow 3)^{*}$ (by the Equations (10), (12) and (14).

\section{Problem Calculations}

In a good reputed organization with four fast moving gadgets, Purchase cost is $(15,000,12000,9000$, 
6000), 40\% marginal profit and ordering cost is Rs. 0.5 per unit price, holding cost Rs. 2 per unit hold, selling value of four categories are in stages with $D_{f}(\alpha, \beta, \gamma, \delta)=(5,10,15,20) \%$ for defectives, loosing amount of $25 \%$ depreciation cost in the repairing process and $93 \%$ of losing value in scrap process. $0.53 \%$,
$2.7 \%, 1.33 \%, 4 \%$ and $2 \%$ are the testing, labor, additional materials, scrap and clearing charges respectively. Here, $(4000,3000,2000,1000)$ are the number of gadget and guaranty, warranty and scrap periods are 90, 30 and 30 days respectively. Find the total profit.

\subsection{For Down Process Table 1.}

Table 1. For down process

\begin{tabular}{|c|c|c|c|c|c|c|c|c|c|c|}
\hline & $\tilde{r}$ & $\tilde{P_{c t}}$ & $\widetilde{P_{C}}$ & $\tilde{O}$ & $\widetilde{H}$ & {$[\widehat{T D N}]$} & $\mathbf{N}^{\tilde{r}} \tilde{r}$ & $\widetilde{\mathrm{SV}_{\mathrm{G}}}$ & {$\left[R_{e v_{1} D} \bar{D}\right]$} & {$\left[\widehat{P_{1} \overline{D N}}\right]$} \\
\hline \multirow{4}{*}{$\alpha$} & 4000 & 15000 & 60000000 & 2000 & 360000 & 362000 & 3800 & 25000 & 95000000 & 34638000 \\
\hline & 3000 & 12000 & 36000000 & 1500 & 270000 & 271500 & 2850 & 20000 & 57000000 & 20728500 \\
\hline & 2000 & 9000 & 18000000 & 1000 & 180000 & 181000 & 1900 & 15000 & 28500000 & 10319000 \\
\hline & 1000 & 6000 & 6000000 & 500 & 90000 & 90500 & 950 & 10000 & 9500000 & 3409500 \\
\hline \multirow{4}{*}{$\beta$} & 4000 & 15000 & 60000000 & 2000 & 360000 & 362000 & 3600 & 25000 & 90000000 & 29638000 \\
\hline & 3000 & 12000 & 36000000 & 1500 & 270000 & 271500 & 2700 & 20000 & 54000000 & 17728500 \\
\hline & 2000 & 9000 & 18000000 & 1000 & 180000 & 181000 & 1800 & 15000 & 27000000 & 8819000 \\
\hline & 1000 & 6000 & 6000000 & 500 & 90000 & 90500 & 900 & 10000 & 9000000 & 2909500 \\
\hline \multirow{4}{*}{$\gamma$} & 4000 & 15000 & 60000000 & 2000 & 360000 & 362000 & 3400 & 25000 & 85000000 & 24638000 \\
\hline & 3000 & 12000 & 36000000 & 1500 & 270000 & 271500 & 2550 & 20000 & 51000000 & 14728500 \\
\hline & 2000 & 9000 & 18000000 & 1000 & 180000 & 181000 & 1700 & 15000 & 25500000 & 7319000 \\
\hline & 1000 & 6000 & 6000000 & 500 & 90000 & 90500 & 850 & 10000 & 8500000 & 2409500 \\
\hline \multirow{4}{*}{$\delta$} & 4000 & 15000 & 60000000 & 2000 & 360000 & 362000 & 3200 & 25000 & 80000000 & 19638000 \\
\hline & 3000 & 12000 & 36000000 & 1500 & 270000 & 271500 & 2400 & 20000 & 48000000 & 11728500 \\
\hline & 2000 & 9000 & 18000000 & 1000 & 180000 & 181000 & 1600 & 15000 & 24000000 & 5819000 \\
\hline & 1000 & 6000 & 6000000 & 500 & 90000 & 90500 & 800 & 10000 & 8000000 & 1909500 \\
\hline
\end{tabular}

\subsubsection{Graphical Representation: 1}

\section{$\alpha, \beta, \gamma, \delta$ in Down process}

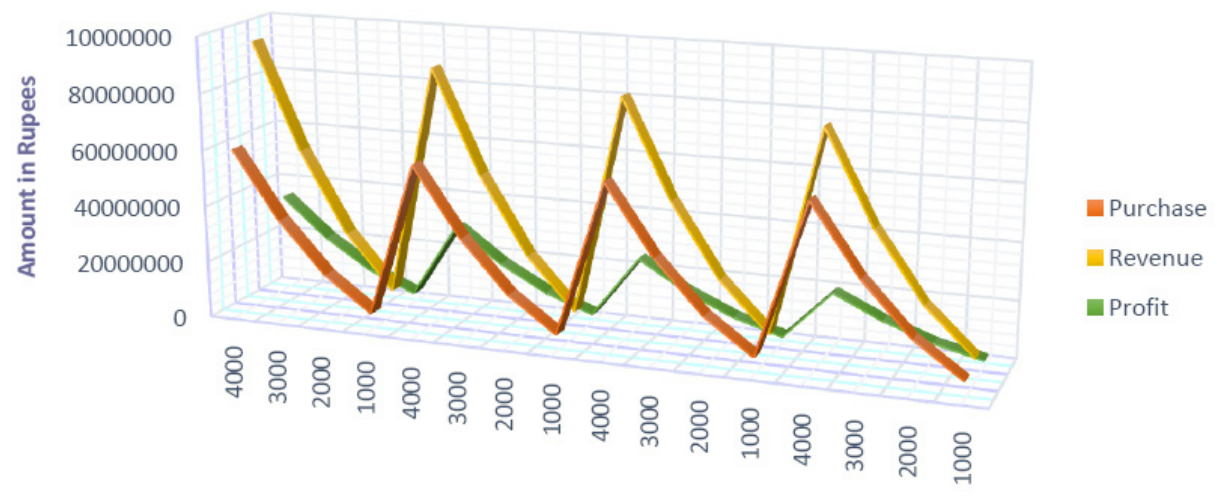




\subsection{Up Process Table 2.}

Table 2. Up process

\begin{tabular}{|c|c|c|c|c|c|c|c|c|c|c|c|}
\hline & $\tilde{r}$ & $N r^{*}$ & $\widetilde{H}$ & $\widetilde{\mathrm{SV}_{\mathrm{D}}}$ & $\mathrm{R}_{1}$ & $\mathrm{R}_{2}$ & $\mathrm{R}_{3}$ & $\left(R_{1}+R_{2}\right) \widetilde{r}+R_{3}(a)$ & {$[\widetilde{T U P}]$} & {$\left[R_{Q \vee 2} \bar{U} P\right]$} & {$\left[\widetilde{P_{2} U P}\right]$} \\
\hline \multirow{4}{*}{$a$} & 200 & 160 & 6000 & 18750 & 20000 & 100000 & 40000 & 160000 & 166000 & 3000000 & 2834000 \\
\hline & 150 & 120 & 4500 & 15000 & 15000 & 75000 & 30000 & 120000 & 124500 & 1950000 & 1825500 \\
\hline & 100 & 80 & 3000 & 11250 & 10000 & 50000 & 20000 & 80000 & 83000 & 900000 & 817000 \\
\hline & 50 & 40 & 1500 & 7500 & 5000 & 25000 & 10000 & 40000 & 41500 & 300000 & 258500 \\
\hline \multirow{4}{*}{$\beta$} & 400 & 320 & 12000 & 18750 & 40000 & 200000 & 80000 & 320000 & 332000 & 6000000 & 5884000 \\
\hline & 300 & 240 & 9000 & 15000 & 30000 & 150000 & 60000 & 240000 & 249000 & 3600000 & 3351000 \\
\hline & 200 & 160 & 6000 & 11250 & 20000 & 100000 & 40000 & 160000 & 166000 & 1800000 & 1634000 \\
\hline & 100 & 80 & 3000 & 7500 & 10000 & 50000 & 20000 & 80000 & 83000 & 600000 & 517000 \\
\hline \multirow{4}{*}{$\gamma$} & 600 & 480 & 18000 & 18750 & 60000 & 300000 & 120000 & 480000 & 498000 & 9000000 & 8502000 \\
\hline & 450 & 360 & 13500 & 15000 & 45000 & 225000 & 90000 & 360000 & 373500 & 5400000 & 5026500 \\
\hline & 300 & 240 & 9000 & 11250 & 30000 & 150000 & 60000 & 240000 & 249000 & 2700000 & 2451000 \\
\hline & 150 & 120 & 4500 & 7500 & 15000 & 75000 & 30000 & 120000 & 124500 & 900000 & 775500 \\
\hline \multirow{4}{*}{$\delta$} & 800 & 640 & 24000 & 18750 & 80000 & 400000 & 160000 & 640000 & 664000 & 12000000 & 11336000 \\
\hline & 600 & 480 & 18000 & 15000 & 60000 & 300000 & 120000 & 372000 & 390000 & 7200000 & 6702000 \\
\hline & 400 & 320 & 12000 & 11250 & 40000 & 200000 & 80000 & 320000 & 332000 & 3600000 & 3448000 \\
\hline & 200 & 160 & 6000 & 7500 & 20000 & 100000 & 40000 & 160000 & 166000 & 1200000 & 1034000 \\
\hline
\end{tabular}

\subsection{Graphical Representation: 2}

\section{$\alpha, \beta, \gamma, \delta$ in Up process}

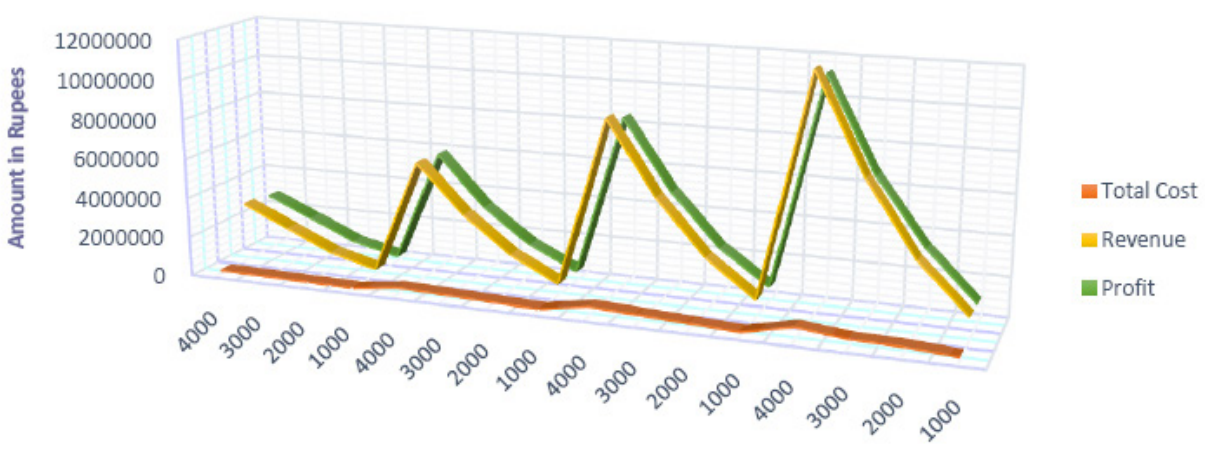




\subsection{Scrap/Clearing Process Table 3.}

Table 3. Scrap/clearing process

\begin{tabular}{|c|c|c|c|c|c|c|c|c|}
\hline & $\widetilde{r} \cdot$ & $\widetilde{H}$ & $\overline{\mathrm{SV}}$ & $\mathrm{R}_{4}$ & $\mathrm{C}_{\mathrm{c}}$ & {$[\overline{T R C}]$} & {$\left[R_{e v \mathrm{~s}} R \mathrm{c}\right]$} & {$\left[\widetilde{P_{3} \overline{R C}}\right]$} \\
\hline \multirow{4}{*}{$\alpha$} & 40 & 1200 & 1250 & 2000 & 1000 & 4200 & 50000 & 45800 \\
\hline & 30 & 900 & 1000 & 1500 & 750 & 3150 & 30000 & 26850 \\
\hline & 20 & 600 & 750 & 1000 & 500 & 2100 & 15000 & 12900 \\
\hline & 10 & 300 & 500 & 500 & 250 & 1050 & 5000 & 3950 \\
\hline \multirow{4}{*}{$\beta$} & 80 & 2400 & 1250 & 4000 & 2000 & 8400 & 100000 & 91600 \\
\hline & 60 & 1800 & 1000 & 3000 & 1500 & 6300 & 60000 & 53700 \\
\hline & 40 & 1200 & 750 & 2000 & 1000 & 4200 & 30000 & 25800 \\
\hline & 20 & 600 & 500 & 1000 & 500 & 2100 & 10000 & 7900 \\
\hline \multirow{4}{*}{$\gamma$} & 120 & 3600 & 1250 & 6000 & 3000 & 12600 & 150000 & 137400 \\
\hline & 90 & 2700 & 1000 & 4500 & 2250 & 9450 & 90000 & 80550 \\
\hline & 60 & 1800 & 750 & 3000 & 1500 & 6300 & 45000 & 38700 \\
\hline & 30 & 900 & 500 & 1500 & 750 & 3150 & 15000 & 11850 \\
\hline \multirow{4}{*}{$\delta$} & 160 & 4800 & 1250 & 8000 & 4000 & 16800 & 200000 & 183200 \\
\hline & 120 & 3600 & 1000 & 6000 & 3000 & 12600 & 120000 & 107400 \\
\hline & 80 & 2400 & 750 & 4000 & 2000 & 8400 & 60000 & 51600 \\
\hline & 40 & 1200 & 500 & 2000 & 1000 & 4200 & 20000 & 15800 \\
\hline
\end{tabular}

\subsection{Graphical Represenatation: 3}

\section{$\alpha, \beta, \gamma, \delta$ in Scrap process}

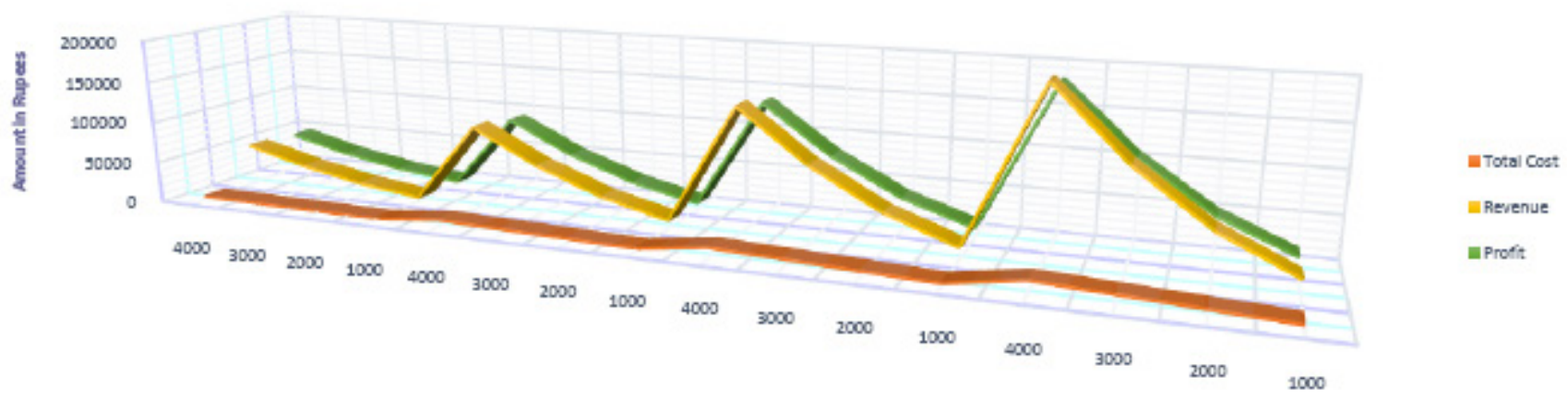




\subsection{Comparison of Lot Size and Profit with Down, Up and Scrap Process. Table 4.}

Table 4. Comparison of lot size and profit with Down, Up and Scrap process

\begin{tabular}{|c|c|c|c|c|c|}
\hline Lot Size & $\mathrm{D}_{\mathrm{f}}$ & $\tilde{\boldsymbol{D P}}$ & $\tilde{\boldsymbol{U P}}$ & $\tilde{\boldsymbol{S P}}$ & Profit \\
\hline \multirow{2}{*}{ Lot 1} & $\alpha$ & 34638000 & 3000000 & 45800 & 37683800 \\
\cline { 2 - 6 } & $\beta$ & 29638000 & 6000000 & 91600 & 35729600 \\
\cline { 2 - 6 } & $\gamma$ & 24638000 & 9000000 & 137400 & 33775400 \\
\cline { 2 - 6 } & $\delta$ & 19638000 & 12000000 & 183200 & 31821200 \\
\hline \multirow{3}{*}{ Lot 2 } & $\alpha$ & 20728500 & 1950000 & 26850 & 22705350 \\
\cline { 2 - 6 } & $\beta$ & 17728500 & 3600000 & 53700 & 21382200 \\
\cline { 2 - 6 } & $\gamma$ & 14728500 & 5400000 & 80550 & 20209050 \\
\cline { 2 - 6 } & $\delta$ & 11728500 & 7200000 & 107400 & 19035900 \\
\hline \multirow{2}{*}{ Lot 3 } & $\alpha$ & 10319000 & 900000 & 12900 & 11231900 \\
\cline { 2 - 6 } & $\beta$ & 8819000 & 1800000 & 25800 & 10644800 \\
\cline { 2 - 6 } & $\gamma$ & 7319000 & 2700000 & 38700 & 10057700 \\
\cline { 2 - 6 } & $\delta$ & 5819000 & 3600000 & 51600 & 9470600 \\
\hline \multirow{5}{*}{ Lot 4 } & $\alpha$ & 3409500 & 300000 & 3950 & 3713450 \\
\cline { 2 - 6 } & $\beta$ & 2909500 & 600000 & 7900 & 3517400 \\
\cline { 2 - 6 } & $\gamma$ & 2409500 & 900000 & 11850 & 3321350 \\
\hline & $\delta$ & 1909500 & 1200000 & 15800 & 3125300 \\
\hline
\end{tabular}

\subsubsection{Graphical Representation. 4}

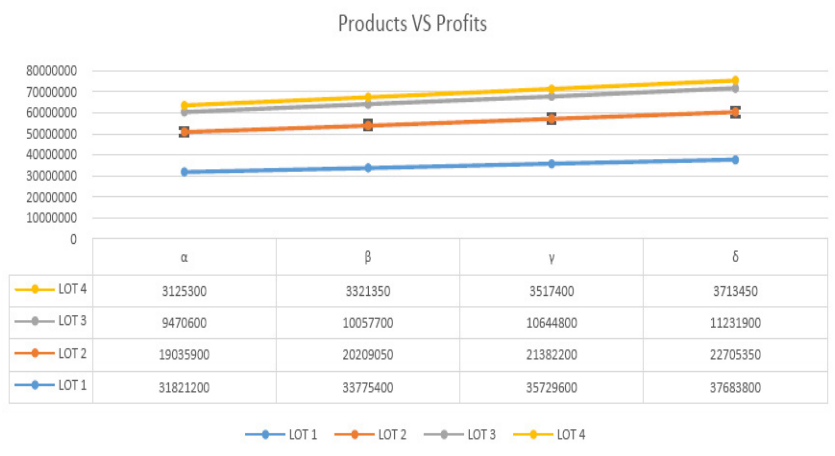

\section{Conclusion}

Loss of money occurs due to defective goods sometimes reaches assumed partial loss while others considered full loss. In real life, all buyers wait or go the next product. Then it is called total loss otherwise all the buyers will left the system however, in certain situations some customers will be able to wait for the next order in order to satisfy their demands during the warranty period (or) guaranty period while others so not wish to or cannot wait hence, they meet their demands from the other sources (partial loss case). From the Table 4 heavy loss can be avoided with Up process and addition to that scrap revenue also filled the gap of the gain percentage. Moreover, graphical representations shows that complete loss reaches to profit percentage.

From the Tables 1, 2, 3 and 4, it can be observed that,

- Revenue decreases when in the Down Process.

- Profit is moderate when in the Down process.

- Good quality lot size decreases when in down process but profit percentage increases.

- Again lot size decreases in the scrap process but total profit percentage increases slightly.

- Selling price is stable when Down process.

- Selling price decreases when in Up process.

- Selling price highly decreases in scrap process.

- Profit increases when in the Up process instead of loss.

- Partial loss increases when in the scrap process instead of full loss.

- Revenue is increases when in Up, Down and scrap process with partial loss.

- Ordering and holding costs are same in all tables with respect to lot size but profit percentage is varies due to defectives and losing the selling price and brand value.

A fuzzy inventory model for demand with constant selling price, ordering cost and holding cost has been developed with fuzzy sense. Trapezoidal fuzzy models are found for profit/loss. A numerical example is also given in support the theory. A future research is to extend the model under uncertain demand with different profit margins.

\section{Acknowledgements}

The authors are indebted to the Professors and the referees for their helpful and constructive comments that improved the presentation of the paper. Research was affiliated by the University of Madras in India, Ph.D.

\section{References}

1. Hsu WKK. Optimal inventory model with fuzzy perfective rate, demand rate and purchasing cost under immediate return for defective items. International Journal of Innovative Computing Information and Control. 2012; 8(4):2583-98. 
2. Rajoria YK, Singh SR, Saini S. An inventory model for decaying item with ramp demand pattern under inflation and partial backlogging. Indian Journal of Science and Technology. 2015 Jun; 8(12):1-6.

3. Hsu WKK, Yu HF. Economic order quantity model with immediate return for defective items. ICIC Express Letters. 2011; 5(7):2215-20.

4. Vikram V, Ajay T, Chandra S, Malik AK. A trade credit inventory model with multivariate demand for non-instantaneous decaying products. Indian Journal of Science and Technology. 2016 Apr; 9(15):1-6.

5. Salameh MK, Jaber MY. Economic order quantity model for imperfect quality. International Journal of Production and Economics. 2000; 64(1-3):59-64.

6. Shahraki MR, Shahraki A, Javdan N. Developing an inventory mathematical model with deterioration variables for discounted stochastic goods. Indian Journal of Science and Technology. 2014 Jan; 7(11):1750-5.

7. Hsieh $\mathrm{CH}$. Optimization of fuzzy inventory models under fuzzy demand and fuzzy lead time. Tamsui Oxford Journal of Management Sciences. 2004; 20(10):21-36.

8. Bhagoria M, Sadiwala CM, Khare VK. Multilevel inventory techniques for minimizing cost-a case study. Indian Journal of Science and Technology. 2010 Jun; 3(6):693-5.

9. Dubois D, Prade H. Fuzzy sets and systems: Theory and applications. New York: Academic Press; 1980,

10. Sommer G. Fuzzy inventory scheduling applied systems and cybernetics. Applied Systems and Cybernetics. G. Lasker, editor. Academic Press, New York. 1981; 6:3052-60.

11. Sharma S, Singh SR. An inventory model for decaying items, considering multivariate consumption rate with partial backlogging. Indian Journal of Science and Technology. 2013 Jul; 6(7):4870-80.

12. Tayal S, Singh SR, Sharma R. A multi item inventory model for deteriorating items with expiration date and allowable shortages. Indian Journal of Science and Technology. 2014; 7(4):463-71.

13. Zuikov S. A literature review on models of inventory management under uncertainty. Business systems and Economics. 2015; 5(1):26-35.

14. Aarya DD, Kumar M. Supply chain model with ramp type demand under planning horizon. Indian Journal of Science and Technology. 2015; 8(15):1-8.

15. Roy AR, Dutta P, Chakraborty D. An inventory model for single period products with reordering opportunities under fuzzy demand. An International Journal of Computers and Mathematics with Applications. 2007; 53(10):1502-17.

16. Nalina V, Jeeva M. K-release inventory model in manpower planning. Indian Journal of Science and Technology. 2010 Jul; 3(7):727-32.

17. Dutta D, Kumar P. A partial backlogging inventory model for deteriorating items with time varying demand and holding cost. Croatian Operational Research review. 2015; 6(2):321-34.

18. Chaudhary RR, Sharma V. A model for Weibull deteriorate items with price dependent demand rate and inflation. Indian Journal of Science and Technology. 2015 May; 8(10):975-81.

19. Mandal NK. Fuzzy economic order quantity model with ranking fuzzy number cost parameters. Yugaslav Journal of Operations Research. 2012; 22(2):247-64.

20. Nailwal KK, Gupta D, Sharma S. Two stage flow shop scheduling under fuzzy environment. Indian Journal of Science and Technology. 2015; 8(16):1-8.

21. Banerjee S, Roy TK. Arithmetic operations on generalized trapezoidal fuzzy number and its applications. Turkish Journal of Fuzzy Systems. 2012; 3(1):16-44.

22. Gani AN, Mohamed Assarudeen SN. A new operation on triangular fuzzy number for solving fuzzy linear programming problem. Applied Mathematical Sciences. 2012; 6(11):525-32.

23. Mishra N, Soni JK. An EOQ inventory model with fuzzy deterioration rate and finite production rate. IOSR Journal of Mathematics. 2012; 4(4):1-9.

24. Pravin Kumar M, Vijayachitra S. Neuro fuzzy technique for steel process modeling. Indian Journal of Science and Technology. 2015 Sep; 8(24):1-7.

25. Manimaran S, Ananthanarayanan M. A study on comparison between fuzzy assignment problems using trapezoidal fuzzy numbers with average method. Indian Journal of Science and Technology. 2012 Apr; 5(4):2610-3.

26. Karamizadeh F, Zolfagharifar SA. Using the clustering algorithms and rule-based of data mining to identify affecting factors in the profit and loss of third party insurance, insurance company auto. Indian Journal of Science and Technology. 2016 Feb; 9(7):1-9.

27. Heidarpoor F, Shahrivar FS. Unsystematic risk and internal control quality impact on the earning quality by using volatility profits index in Tehran Stock Exchange. Indian Journal of Science and Technology. 2015 Jun; 8(11):1-6. 